\title{
A Case of Mucosa-Associated Lymphoid Tissue Lymphoma (MALToma) That Developed From Lungs and Stomach
}

\author{
Hiroshi Ono ${ }^{\mathrm{a}, \mathrm{b}, \mathrm{d}}$, Yasuyuki Taniguchi ${ }^{\mathrm{a}, \mathrm{b}}$, Takaki Shimada ${ }^{\mathrm{c}}$, Akihiko Gemma ${ }^{\mathrm{b}}$
}

\begin{abstract}
The case concerns a 52-year-old male who presented at our hospital because of abnormal chest X-ray findings following a group medical examination. On the computed tomography (CT) image, there was an infiltrative shadow with bronchiectasis manifest in the right lower lobe, a right pleural effusion (PE), densities on bronchovascular bundles elsewhere in the lungs and clear thickening of the gastric wall. In the PE, we detected cells reminiscent morphologically of malignant lymphoma and with a chromosomal abnormality, and a gene rearrangement of the B-cell. By trans-bronchial brushing, we detected cells that were similar to those in the PE in the right lower lobe, and the findings of transbronchial lung biopsies (TBLB) and gastric biopsies were both compatible with mucosa-associated lymphoid tissue lymphoma (MALToma). Thus, we diagnosed this as a case of MALToma which developed from the lungs and the stomach, and suggest that MALToma should be considered in the differential diagnosis of lung disease.
\end{abstract}

Keywords: Mucosa-associated lymphoid tissue lymphoma (MALToma); Pleural effusion; Bronchiectasis

\section{Introduction}

Mucosa-associated lymphoid tissue lymphoma (MALToma) is a low grade B-cell lymphoma which was proposed by Isaacson and Wright in 1983 [1]. In the latest WHO classifica-

Manuscript accepted for publication November 17, 2010

\footnotetext{
${ }^{\mathrm{a}}$ Jiseikai-Tojun Hospital, Japan

${ }^{\mathrm{b}}$ Division of Pulmonary Medicine, Infectious Disease, and Oncology, Department of Internal Medicine, Nippon Medical School, Japan ${ }^{\mathrm{c}}$ Division of Clinical Oncology/Hematology, Department of Internal Medicine, The Jikei University School of Medicine, Japan ${ }^{\mathrm{d}}$ Corresponding author: h-ono@nms.ac.jp
}

doi: $10.4021 / \mathrm{jmc} 87 \mathrm{w}$ tion, it belongs to the extra-nodal marginal zone lymphoma. This lymphoma develops from lungs, intestinal tract, salivary gland, thyroid gland, and prostate, etc. The incidence of MALToma that develops from lungs is rare, about $0.1 \%$ of pulmonary malignant tumors, although it represents about $90 \%$ of non-Hodgkin's lymphomas of a pulmonary origin [2]. Thus, it should be considered in the differential diagnosis of pulmonary diseases. We experienced a case with right pleural effusion (PE) and collapse, with bronchiectasis in the right lower lobe of the lungs, and initially we suspected a pulmonary infectious disease. However, the case was subsequently diagnosed by pathology and genetics as a MALToma which had developed from the lungs and the stomach, and we report the case because of its importance in MALToma diagnosis, especially when based on imaging.

\section{Case Report}

The patient was a 52-year-old male who was unemployed and had bipolar disorder and had attended a psychiatry hospital for about 40 years, and presented at Jiseikai-Tojun Hospital in September 2009 because of dyspnea and an abnormal shadow on a chest X-ray following a routine medical examination. He had no allergy and no inhalation history reported particularly. He had been smoking 1.5 packs of cigarettes per day for about 20 years (20y.o. - 40y.o.), but had no history of alcohol problems. The result of the physical examination at initial diagnosis were as follows: body temperature 35.6 degrees centigrade, pulse 72 beats per minute regular, blood pressure 154/90 $\mathrm{mmHg}$, and oxygen saturation $91 \%$. By auscultation, no abnormal sound was heard on his thorax, but on the right side, sounds were attenuated. The results of the blood examination, the urinalysis, the urinary sediment, and the PE findings are shown in Table 1. White blood cells (WBC) were increased, and although the ratio of neutrophils was increased, immature WBC were not present. Serum levels of interleukin-2 receptor were high. M-protein of IgM- $\kappa$ and $\mathrm{IgG}-\kappa$ type was detected on immuno-electrophoresis examination, suggestive of macroglobulinemia. No abnormal findings were reported in the urinalysis and urinary sediment. Pseudomonas aeruginosa was detected $(3+)$ in the 
Table 1. Results of Blood Examination, Urinalysis, Urinary Sediment and PE Findings

\begin{tabular}{|c|c|c|c|c|c|c|c|}
\hline$(\mathrm{CBC})$ & & (BBC) & & & & (Urinalysis) & \\
\hline WBC & $19500 / \mathrm{mm}$ & GOT & $14 \mathrm{U} / 1$ & $\operatorname{IgA}$ & $115 \mathrm{mg} / \mathrm{dl}$ & $\mathrm{PH}$ & 6 \\
\hline $\mathrm{RBC}$ & $448 \times 10^{4} / \mathrm{mm}$ & GPT & $10 \mathrm{U} / 1$ & $\operatorname{IgG}$ & $2022 \mathrm{mg} / \mathrm{dl}$ & Protein & - \\
\hline $\mathrm{Hb}$ & $14.2 \mathrm{~g} / \mathrm{dl}$ & LDH & $243 \mathrm{U} / 1$ & $\operatorname{IgM}$ & $570 \mathrm{mg} / \mathrm{dl}$ & Sugar & - \\
\hline $\mathrm{Ht}$ & $41.7 \%$ & $\mathrm{TP}$ & $8.4 \mathrm{~g} / \mathrm{dl}$ & $\operatorname{IgE}$ & 13 & Occult blood & \pm \\
\hline $\mathrm{MCV}$ & 93 & T-cho & $115 \mathrm{mg} / \mathrm{dl}$ & IEP & IgM-к Type & BJP & - \\
\hline $\mathrm{MCH}$ & 31.7 & TG & $69 \mathrm{mg} / \mathrm{dl}$ & & IgG-к Type & -sediment- & \\
\hline $\mathrm{MCHC}$ & $34.1 \%$ & HDL-cho & $30 \mathrm{mg} / \mathrm{dl}$ & sIL-2R & $1740 \mathrm{U} / \mathrm{dl}$ & $\mathrm{RBC}$ & 1-4/HPF \\
\hline Plt & $41.7 \times 10^{4} / \mathrm{mm}$ & $\mathrm{Na}$ & $133 \mathrm{mEq}$ & & & WBC & $<1 / \mathrm{HPF}$ \\
\hline (Hemogram) & & K & $4.6 \mathrm{mEq}$ & (ABG) & & & \\
\hline Stab & $3 \%$ & $\mathrm{Cl}$ & $102 \mathrm{mEq}$ & $\mathrm{PH}$ & 7.454 & (PE) & \\
\hline Seg & $86 \%$ & Glu & $110 \mathrm{mg} / \mathrm{dl}$ & $\mathrm{PCO} 2$ & $36.2 \mathrm{mmHg}$ & $\mathrm{PH}$ & 7.2 \\
\hline Lympho & $4.5 \%$ & CRP & $4.73 \mathrm{mg} / \mathrm{dl}$ & $\mathrm{PO} 2$ & $73 \mathrm{mgHg}$ & protein & $6.0 \mathrm{~g} / \mathrm{dl}$ \\
\hline Mono & $4 \%$ & CEA & $1.7 \mathrm{ng} / \mathrm{dl}$ & $\mathrm{HCO}^{3-}$ & $24.8 \mathrm{mmol} / 1$ & $\mathrm{ADA}$ & $163 \mathrm{IU} / \mathrm{L}$ \\
\hline Eosino & $1.5 \%$ & $\mathrm{SCC}$ & $0.6 \mathrm{ng} / \mathrm{dl}$ & $\mathrm{BE}$ & $1.3 \mathrm{mmol} / 1$ & Cell & neutrophil 12\% \\
\hline Baso & $1 \%$ & NSE & $8.6 \mathrm{ng} / \mathrm{dl}$ & $\mathrm{SaO}_{2}$ & $95.4 \%$ & Fractionation & lymphocyte $88 \%$ \\
\hline
\end{tabular}

(CBC), (complete blood count); WBC, white blood cell; RBC, red blood cell; Hb, hemoglobin; $\mathrm{Ht}$, hematocrit; Plt, blood platelet; (HG), hemogram; stab, stab cell; seg, segmented cell; lympho, lymphocyte; mono, monocyte; eosino, eosinophil; baso, basophil; BBC, blood biochemistry; AST, aspartate aminotransferase; ALT, alanine aminotransferase; LDH, lactate dehydrogenase; BUN, blood urea nitrogen; Cre, creatinine; UA, uric acid; TP, total protein; T-cho, total cholesterol; TG, triglyceride; Glu, glucose; CRP, C-reactive protein; CEA, carcinoembryonic antigen; SCC, squamous cell carcinoma related antigen; NSE, neuron specific enolase; IgG, immunogloblin G; IgM , immunogloblin M; IgE, immunoglobulin E; IEP, Immuno-electrophoresis; SLI-2R, serum interleukin II receptor; (ABG), (arterial blood gas analysis); BE, base excess; SaO2, Oxygen saturation; PE, pleural effusion; BJP, Bence-Jones protein; ADA, adenosine deaminase.

sputum culture, and both of the sputum smear and culture of acid-fast bacillus were negative. In the sputum cytology, class I was detected with Papanicolaou staining. The tuberculin test was negative. The findings of standard chest X-ray and chest-abdomen enhanced computed tomography (CT) are shown in Fig. 1. On the chest X-ray, there was a permeable area with multiple cystic lesions in the right lower lobe. On the chest-abdomen enhanced CT, there was an infiltrative shadow with bronchodilatation manifest in the right lower lobe and right PE. In other areas of the lungs, there were multiple, slight papular lesions and small nodular lesions which appeared predominantly in the periphery. A portion of the mediastinal lymph nodes was enlarged. In addition, the gastric wall was notably thickened, and a portion of the lymph nodes of the stomach, on the lesser curvature side, was enlarged.

Following prompt hospitalization, we inserted a tube into the right pleural cavity of the patient. The exudative PE was yellow and a little turbid. In the cell fractionation of the $\mathrm{PE}$, lymphocytes were dominant. Both the bacterial culture and the acid-fast bacillus smear examination of the PE were negative. However, in the cytodiagnosis of the PE, there were many cells that were larger than lymphocytes, which were basophilic after staining with Giemsa solution. Furthermore, the nuclei of these cells were enlarged and had an abnormal outline, and were judged class IV by Papanicolaou staining. These findings led us to suspect myeloma, leukemia, or malignant lymphoma (Fig. 2). However, we felt that leukemia or myeloma could be excluded because there was no evidence of tumor lesions in the findings from the bone marrow biopsy. Therefore, we took additional samples of the patient's PE and preformed a gene rearrangement (GR) test of lymphocyte surface antigen by the polymerase chain reaction (PCR) method, and GR was found of both B-cell (Immune globulin $\mathrm{H}$ ) and T-cell (TRB) origin, although that of B-cell origin was more strongly developed.

We also performed a fibrotic bronchoscopy. Although the observation of the bronchial lumen indicated no bronchial morphologic abnormality, large quantities of purulent sputum were noted, in which a small quantity of Candida glabrata was detected in cultures of the sputum aspirate. In addition, we performed transbronchial brushing cytodiagnosis (TBC) and transbronchial lung biopsy (TBLB) of the lower right lobe. In TBC, cells were detected which were 


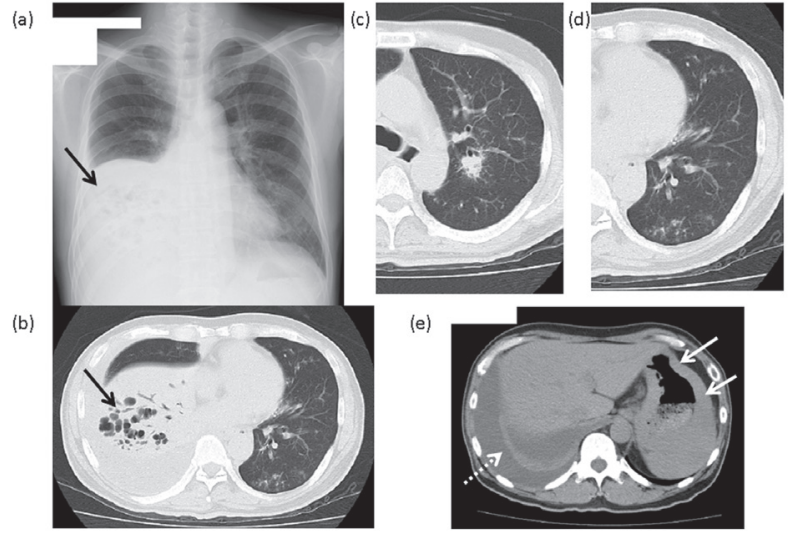

Figure 1. The findings of chest $\mathrm{X}$-ray and computed tomography (CT). (a) Chest X-ray: An area of reduced permeability, with multiple cysts, is visible in the lower right lobe (arrow). (b) Chest CT: An infiltrative shadow with manifest bronchiectasis can be seen in the right lower lobe (arrow). (c) A nodular lesion with an air bronchogram is visible around the center side of the bronchus of the right upper lobe. (d) Multiple ground-glass opacities and granular lesions are visible around the bronchus on the periphery of the left lower lobe. (e) A right pleural effusion (dashed line arrow) and the gastric wall with manifest thickening (arrow) can be seen on this image.

judged class V with Papanicolaou staining, and which were similar to those in the PE (Fig. 3). In TBLB, there were massive lymphoid cellular invasions and lymphoepithelial lesions (LELs), which made us suspect MALToma (Fig. 4). We performed a fibrotic gastroscopy because of the thickened gastric wall, clearly evident on the CT scan. Based on these macroscopic findings of tissue manifestly invaded by lymphoid cells, edema and hyperplasia of the gastric wall of the entire stomach, gastric cancer of Borrmann type IV was suspected. However, based on the biopsies, the specimen was judged histologically as a Group III gastric cancer with manifest lymphoid invasions and LELs, by which MALToma was suspected of both the stomach and the lungs. In ad-
(Papanicolaou staining)

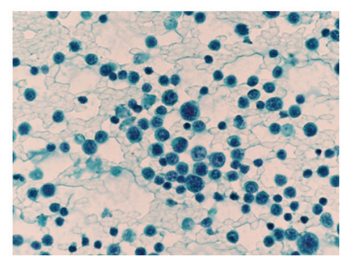

(Giemsa staining)

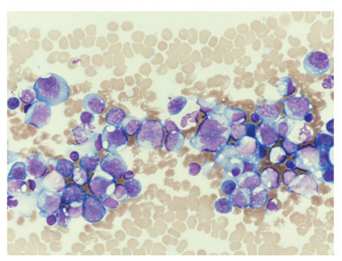

Figure 2. The cytology of the pleural effusion. Large numbers of cells were detected, which were class IV by Papanicolaou staining, larger than lymphocytes, basophilic by Giemsa staining and with cellular nuclei that were enlarged and existed deviating from center. This led us to suspect multiple myeloma, leukemia or malignant lymphoma.
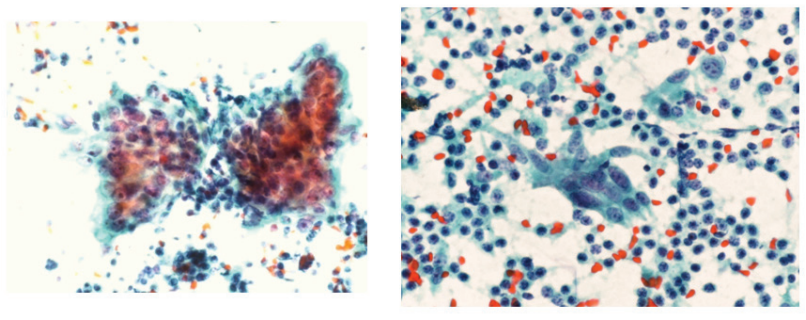

Figure 3. The brush cytology of the bronchus in the right lower lobe. Cells were detected which were class $\mathrm{V}$ by Papanicolaou stain, and similar to those in the pleural effusion.

dition, a Helicobacter pylori (HP) infection was detected via the use of a quick HP diagnostic kit (PyloriTek ${ }^{\circledR}$ ) at the time of the gastroscopy.

Initially, we had suspected an infectious disease of the lungs, and therefore we had administered antibiotics, starting with SBT/ABPC (6 g/day) and CPFX (0.6 g/day) for one week. This was subsequently changed to SBT/CPZ (2 $\mathrm{g} /$ day), administered for nine days, because of P. aeruginosa detected in the sputum culture. We prescribed clarithromycin, lansoprazole, and amoxicillin for one week to eradicate HP. However, in spite of the treatment, no improvement was seen on the image findings. After the above-mentioned course, the patient was transferred to the Respiratory Internal Medicine Unit of Nippon Medical School Hospital, Tokyo for further tests and treatment.

After the patient's transfer, GR test of lymphocyte surface antigen by the Southern blot (SB) method and a chromosome test on the PE were both performed. As a result, GR of IgH chain in JH gene, which suggested B-cell lympho$\mathrm{ma}$, and a chromosome abnormality of $\mathrm{t}(11 ; 18)(\mathrm{q} 21 ; \mathrm{q} 21.3)$ were detected (Fig. 5). In addition, when CD20 staining was performed on the specimens obtained from the stomach by gastroscopy at Nippon Medical School Hospital, there was a manifest lymphoid invasion, LEL in HE staining, and in CD20 staining, an infiltration by CD20-positive cells which predominantly expressed B-cells (Fig. 6). Although we had confirmed the diagnosis of MALToma based on the abovementioned findings, following the request of the patient and
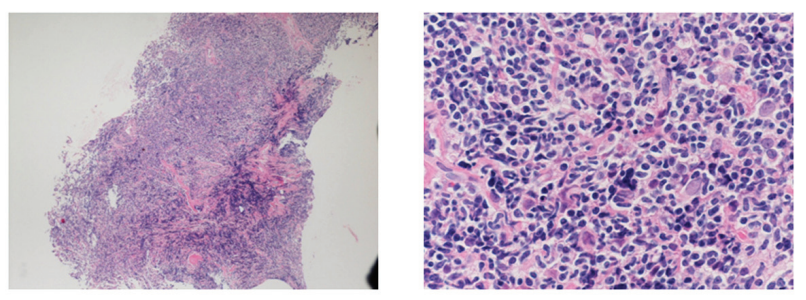

Figure 4. Trans-bronchial lung biopsy of the right lower lobe. A massive invasion of lymphocytes and a lymphoepithelial lesion (LEL) is presented, by which MALToma was suspected. 


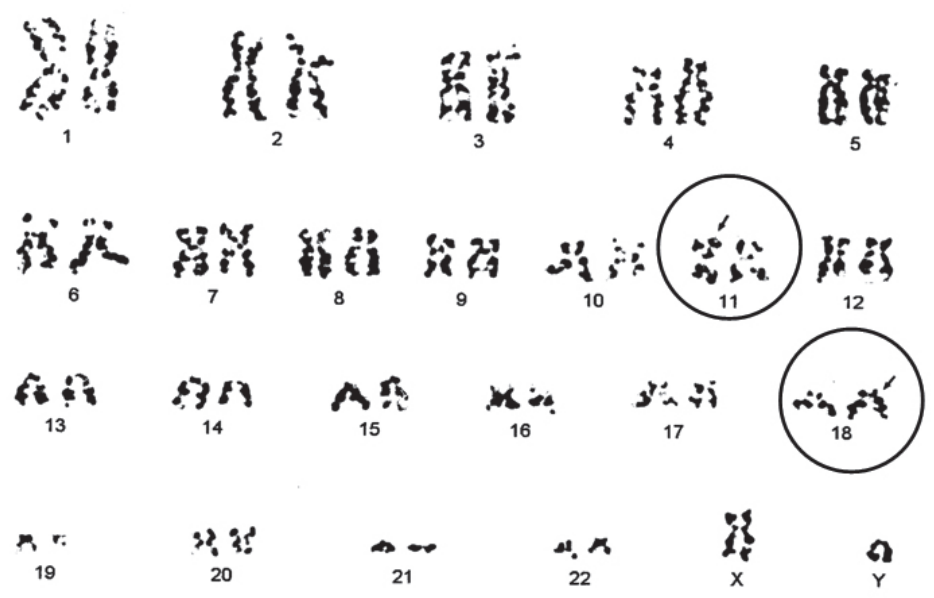

Figure 5. Chromosome analysis of the patient's pleural effusion. Chromosome banding in the pleural effusion, performed in Nippon Medical School Hospital, identified the aberration of $t(11 ; 18)(q 21 ; q 21.3)$.

his family, the patient was transferred a second time to the Unit of Internal Medicine of Hematology and Oncology in Jikei University School of Medicine Hospital, Tokyo. In May 2010, we asked the doctor in charge of the Unit at the hospital for information on the subsequent course of the patient. This doctor reported that a translocation of $t(11 ; 18)$ (q21;q21) had been detected based on the chromosome test of PE performed at this hospital, and that they had administered six courses of R-CHOP therapy to the patient. As a result, the PE had decreased and the hyperplasia of the gastric wall had clearly improved, but the findings of the lower right lobe had remained.

\section{Discussion}

The chest CT of the present case showed an infiltrated lesion in the right lower lobe with a dilated air bronchogram in which the intra-alveolar air spaces had disappeared. The finding could have led us to consider this to be a case of 'destroyed lung', in other words, the structure and function of the lungs appeared to have been permanently destroyed due to repeated inflammation, e.g. because of pulmonary tuberculosis. However, at the initial examination, and based on the imaging, firstly we suspected the present case might

(b)

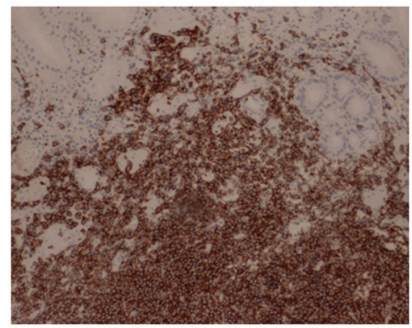

(c)
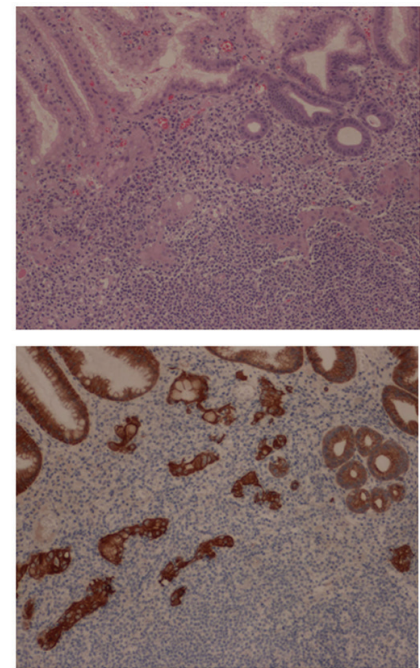

Figure 6. Gastric biopsy in Nippon Medical School Hospital. (a) HE staining: as well as a specimen of TBLB, there was a manifest lymphoid invasion and a lymphoepithelial lesion (LEL). (b) CD20 staining: manifest invasion of CD20 positive $B$ lymphocytes. (c) Keratin staining: destruction of duct configuration by lymphocytes. 
involve an infectious disease, including pulmonary tuberculosis. Ultimately, based on the results of the cytological diagnosis of the PE and the brushing cytological diagnosis of the right lower lobe and TBLB, we obtained a diagnosis of MALToma, which we had not anticipated.

Primary pulmonary MALToma develops from Bronchus-Associated Lymphoid Tissue (BALT), so it is called BALT lymphoma (BALToma). BALT is rarely seen in a healthy adult [3], but grows conspicuously under morbid situations such as follicular bronchitis-bronchiolitis (FBB), diffuse panbronchiolitis (DPB), and bronchiectasis. In the 'background' of cases of FBB, certain types of autoimmune disease, such as rheumatoid arthritis or Sjögren's syndrome, and immune deficiency, such as acquired immunodeficiency syndrome, are often present. On the other hand, it is assumed that chronic inflammation contributes to the outbreak of DPB and BE $[4,5]$.

In the present case, it has been presumed that a chronic infectious disease was the origin of MALToma because of the existence of bronchiectasis. In the case of gastric MALToma, HP (which was seen in the present case) is known to be the causative organism, but in the case of pulmonary MALToma, at present, no species has been established as the causative organism, although a few cases in which Mycobacterium avium, M. tuberculosis, and Epstein-Barr virus were detected have been reported previously [6-8]. In the present case, at the initial examination, $\mathrm{P}$. aeruginosa was detected in the sputum culture, while C. glabrata was detected in the culture of the sputum aspirated by fibrotic bronchoscopy. It is a possibility that repeated episodes of inflammation as a result of these infections had caused MALToma. However, at present, it is not clear how much these bacteria or fungus contribute to the onset of MALT because P. aeruginosa is one of the representative species of bacteria which is contagious in bronchiectasis or secondary DPB, and Candida genus is a fungus which can be present in the buccal capsule of a healthy person. In future, it would be necessary to accumulate cases of MALToma with bronchiectasis and research them bacteriologically for the elucidation of the mechanism, and thus establish more effective treatments for the condition.

In the present case, we obtained specimens from the lungs and stomach and attempted to confirm the suspected MALToma through pathological investigations. However, this was not adequate for arriving at a definitive diagnosis of MALToma, and was supplemented by the presence of GR and chromosomal aberrations in the PE. Both SB and PCR methods can be employed to detect GR. In the present case, initially we could not detect GR with the SB method, but did detect GR of both T- and B-cells using the PCR method. However, when we retried with the SB method, we found GR of the B-cell. Comparing both methods, Ling et al. reported that the PCR method was superior in sensitivity, but inferior in specificity [9], which is the same as the findings in the present case. In the chromosome test of the PE, $\mathrm{t}(11 ; 18)$ (q21;q21.3) and $\mathrm{t}(11 ; 18)(\mathrm{q} 21 ; \mathrm{q} 21)$ were detected on reexamination. The $\mathrm{t}(11 ; 18)(\mathrm{q} 21 ; \mathrm{q} 21), \mathrm{t}(1 ; 14)(\mathrm{p} 22 ; \mathrm{q} 32)$, and $\mathrm{t}(14 ; 18)(\mathrm{q} 32 ; \mathrm{q} 21)$ are known to be translocations of the chromosome related to MALToma. As for $\mathrm{t}(11 ; 18)(\mathrm{q} 21 ; \mathrm{q} 21)$, it is detected in $18 \%$ - 53\% cases of MALToma which develop from all loci $[10,11]$. Some MALTomas are accompanied by macroglobulinemia [12], and for many cases of gastric MALToma, antibiotic therapy for HP is ineffective [10]. The present case was in agreement with earlier reports on both these points.

Several studies which have reported on the findings of chest imaging in MALToma, support the observation that consolidation is present with the highest frequency and, in addition, there are nodular lesions, ground-glass opacity, tree-in-bud sign, bronchiectasis, and reticular lesions, etc. $[11,13,14]$. Bae and coworkers classified the findings of chest CT in BALToma into four patterns, namely solitary nodule with consolidation, multiple nodular lesion with consolidation, bronchiolitis with bronchiectasis, and diffuse interstitial pneumonia [15]. It is difficult to arrive at a diagnosis of MALToma from an infiltrated lesion with manifest bronchiectasis of one entire lobe like the right lower lobe in the present case by recalling conventional chest imaging patterns of MALToma. In addition, there are few case reports of MALToma with PE [16]. Therefore, the present case with the chest imaging findings mentioned above is rare in MALToma. However, when we paid attention to other areas of the lung apart from the right lower lobe, especially in the left lung, we could see ground-glass opacity and tree-in-bud sign all over the left lung, as well as a solitary nodule with consolidation in the left upper lobe, which agree with conventional reports and were strongly suggestive of MALToma. The present case seems to suggest that when we diagnose a disease by chest imaging, we tend to pay attention only to the image findings that show the most remarkable change, and that such findings can in fact rather complicate a differential diagnosis because they often show a terminal state of the disease. Instead, an area that shows only a slight change of density expresses the character of the disease, and can make a differential diagnosis easier.

\section{Acknowledgments}

We are graceful to Dr. Hiroshi Hano, professor of the unit of pathology at Jikei University School of Medicine, for the considerate guidance about pathologic findings.

\section{References}

1. Isaacson $\mathrm{P}$, Wright DH. Malignant lymphoma of mucosa-associated lymphoid tissue. A distinctive type of B- 
cell lymphoma. Cancer 1983;52(8):1410-1416.

2. Fiche M, Caprons F, Berger F, Galateau F, Cordier JF, Loire R, Diebold J. Primary pulmonary non-Hodgkin's lymphomas. Histopathology 1995;26(6):529-537.

3. Tschernig T, Pabst R. Bronchus-associated lymphoid tissue (BALT) is not present in the normal adult lung but in different diseases. Pathobiology 2000;68(1):1-8.

4. Sato A, Hayakawa H, Uchiyama H, Chida K. Cellular distribution of bronchus-associated lymphoid tissue in rheumatoid arthritis. Am J Respir Crit Care Med 1996;154(6 Pt 1):1903-1907.

5. Sato A, Chida K, Iwata M, Hayakawa H. Study of bronchus-associated lymphoid tissue in patients with diffuse panbronchiolitis. Am Rev Respir Dis 1992;146(2):473478.

6. Gaur S, Trayner E, Aish L, Weinstein R. Bronchus-associated lymphoid tissue lymphoma arising in a patient with bronchiectasis and chronic Mycobacterium avium infection. Am J Hematol 2004;77(1):22-25.

7. Inadome $\mathrm{Y}$, Ikezawa T, Oyasu R, Noguchi M. Malignant lymphoma of bronchus-associated lymphoid tissue (BALT) coexistent with pulmonary tuberculosis. Pathol Int 2001;51(10):807-811.

8. Tao J, Kahn L. Epstein-Barr virus-associated highgrade B-cell lymphoma of mucosal-associated lymphoid tissue in a 9-year-old Boy. Arch Pathol Lab Med 2000;124(10):1520-1524.

9. Ling FC, Clarke CE, Corbett WE, Lillicrap DP. Sensitivity of PCR in detecting monoclonal B cell proliferations. J Clin Pathol 1993;46(7):624-627.
10. Auer IA, Gascoyne RD, Connors JM, Cotter FE, Greiner TC, Sanger WG, Horsman DE. $t(11 ; 18)(q 21 ; q 21)$ is the most common translocation in MALT lymphomas. Ann Oncol 1997;8(10):979-985.

11. Ott G, Katzenberger T, Greiner A, Kalla J, Rosenwald A, Heinrich U, Ott MM, et al. The $t(11 ; 18)(q 21 ; q 21)$ chromosome translocation is a frequent and specific aberration in low-grade but not high-grade malignant non-Hodgkin's lymphomas of the mucosa-associated lymphoid tissue (MALT-) type. Cancer Res 1997;57(18):3944-3948.

12. Ye H, Chuang SS, Dogan A, Isaacson PG, Du MQ. $t(1 ; 14)$ and $t(11 ; 18)$ in the differential diagnosis of Waldenstrom's macroglobulinemia. Mod Pathol 2004;17(9):1150-1154.

13. Bae YA, Lee KS, Han J, Ko YH, Kim BT, Chung MJ, Kim TS. Marginal zone B-cell lymphoma of bronchusassociated lymphoid tissue: imaging findings in 21 patients. Chest 2008;133(2):433-440.

14. King LJ, Padley SP, Wotherspoon AC, Nicholson AG. Pulmonary MALT lymphoma: imaging findings in 24 cases. Eur Radiol 2000;10(12):1932-1938.

15. Lee DK, Im JG, Lee KS, Lee JS, Seo JB, Goo JM, Kim TS, et al. B-cell lymphoma of bronchus-associated lymphoid tissue (BALT): CT features in 10 patients. J Comput Assist Tomogr 2000;24(1):30-34.

16. Ikehara M, Yamaguchi H, Yamamoto T, Komase Y, Sano F, Shinagawa T. [Case of pulmonary MALT lymphoma with follow-up imaging for 10 years]. Nihon Kokyuki Gakkai Zasshi 2008;46(2):146-151. 